\title{
Emmanuelle Sempère, \\ De la merveille à l'inquiétude : le registre du fantastique dans la fiction narrative au XVIII siècle
}

Bordeaux, Presses universitaires de Bordeaux, 2009, 611 p.

\author{
Isabelle Tremblay \\ Collège militaire de Kingston
}

Dans cet ouvrage, Emmanuelle Sempère s'interroge sur les " effets d'inquiétude » (17) que produit la lecture de nombreux textes narratifs afin de définir le «registre» (103) du fantastique au XVIIIe siècle. L'étude apporte un éclairage nouveau aux contes, romans et fictions scientifiques des Lumières souvent perçus comme textes précurseurs de la littérature fantastique du XIXe siècle.

\section{De la superstition au surnaturel}

L'auteure se penche d'abord sur le traitement que subit la superstition et sur la place grandissante qu'occupe le surnaturel 
dans le discours pseudo-scientifique, l'Encyclopédie et le genre romanesque. Dans la première moitié du siècle, les ouvrages traitant de la superstition tendent à l'associer à une pratique marginale et à une «faiblesse intellectuelle » (89), la discréditant ainsi aux yeux de tout catholique cultivé. Avec Augustin Calmet et son Traité des apparitions des esprits et sur les vampires et les revenants (1746) apparaît le souci de renforcer l'autorité du pouvoir surnaturel de la religion, compris comme ce qui est au-dessus de la nature et qui relève de l'ordre divin. Le succès qu'il remporte ainsi que la réédition et la traduction de son ouvrage témoignent de l'intérêt du public pour le surnaturel. La place qu'occupe le surnaturel dans l'Encyclopédie et la difficulté que manifestent les rédacteurs à s'entendre pour le définir sont le signe qu'il suscite une sorte de malaise. Partagés entre les sentiments de rejet et de fascination à son endroit, les romanciers des Lumières font poindre une part d'ambiguïté dans leurs récits. En pleine redéfinition, le genre romanesque se voit conférer la caution du vrai et conjugue la réalité avec le « plaisir de croire » (470). Parce qu'il se définit par rapport à la représentation d'un monde cohérent, c'est-à-dire par rapport à une norme, le fantastique se construit sur un fond de vraisemblable et suscite le sentiment d'inquiétude «à proportion de l'éclatement de la cohérence du monde» (447). C'est pourquoi il trouve dans le roman des Lumières un espace privilégié où se construire. Comme l'espace romanesque se veut autonome par rapport à la réalité, le surnaturel qui s'y voit «poétisé» (91) se confond avec l'étrangeté et devient "hors Dieu» ou plutôt "hors nature » (91). En résulte le fantastique en tant qu'«inscription dans le 
texte des procédures de réception d'un matériau surnaturel » (91). Espace idéal où mettre en scène le " surnaturel expliqué » (94) et la « confrontation dialogique » (95) servant à dénoncer la superstition, le roman accueille aisément le projet rationaliste des Lumières consistant à découvrir la vérité et à se détacher de l'esprit de système, de sorte que le surnaturel sert de couvert à des affirmations dont il était impossible d'envisager la formulation en termes réalistes.

Alors que les théoriciens du fantastique accordent une attention particulière au Diable amoureux (1776) de Jacques Cazotte, dans lequel les romantiques et surréalistes ont vu un tissu de croyances anciennes et de légendes populaires, Emmanuelle Sempère met l'accent sur le fait que ce roman, qui s'affaire à prévenir son public contre la sensualité et l'amour, condamne le surnaturel plutôt qu'il ne lui accorde une dimension exemplaire. Elle retrace avec précision la présence du «féminin démoniaque» (137) dans d'autres textes de Jacques Cazotte et indique que l'originalité du Diable amoureux réside dans «le doute sur la nature de l'apparition » (140), lequel est source de fantastique et produit des débats de l'époque sur la métamorphose du diable. En plus de montrer que la représentation du surnaturel se nourrit du contexte culturel, l'auteure a le mérite d'illustrer en quoi Le Diable amoureux se distingue d'autres romans lorsqu'il fait intervenir la dimension surnaturelle. Toutefois, le rapprochement entre L'Histoire d'une Grecque moderne (1740) de l'abbé Prévost et Le Diable amoureux (1776) de Cazotte se révèle peu convaincant précisément parce qu'il néglige de tenir compte du contexte esthétique fort différent présidant à l'écriture de chacun. En 
alliant Théophé à la figure de la sorcière, l'auteure montre bien que le roman de Prévost met en scène une «lutte de deux paroles » (148), mais échoue à en dégager toutes les nuances. La conclusion que le texte démonologique sert de modèle au genre romanesque, lui fournissant une «énonciation spécifique », un «modèle rhétorique » et un « modèle narratif » (149), aurait gagné à être explicitée par davantage d'exemples.

\section{Du merveilleux au fantasmagorique}

À la fin du Grand Siècle, le merveilleux, qui permet une certaine liberté d'expression, rend possible la double représentation du réel et de l'étrangeté. Le conte de fées, théâtre du merveilleux, se veut donc la peinture des «points d'inquiétude» (162) de l'époque. La figure du monstre constitue le plus important "symptôme de désordre» (164). Au début du siècle des Lumières, la représentation des monstres est codifiée : diverses fonctions leur sont attribuées selon que le texte littéraire possède un programme moral, précieux ou libertin. En passant en revue la figure du monstre telle qu'elle se manifeste dans les contes de Mme d'Aulnoy, de Lubert et de Crébillon, l'auteure dresse une sorte de symbolique du monstre. Pour sa part, la fée, dont l'auteure étudie les pouvoirs, se situe aux confins d'un «naturel merveilleux » et d'une "nature physique» (205). En se substituant au conte merveilleux dans les années 1730 , le conte parodique, qui manifeste l'inquiétude d'une époque incapable de rompre définitivement avec un certain nombre de croyances et de superstition, ouvre la voie à un discours qui a pour objet non pas le surnaturel, mais l'anormalité. Le 
merveilleux est donc mis à distance dans les contes de Crébillon alors que l'enchantement se voit remplacé par l'ennui dans ceux d'Hamilton.

Après le merveilleux, le fantasmagorique, compris comme un «discours des fantasmes» (347), inspire des œuvres à teneur surnaturelle ayant pour fonction $d$ '«abuser l'imagination » (348). Tout comme le conte merveilleux, le récit fantasmagorique met en scène la figure du monstre, qui effraie plus par l'angoisse qui préside à sa création que par son aspect surnaturel. L'emprunt de figures et de motifs issus des merveilleux gothique et oriental contribue à renouveler le conte et répond à l'engouement des lecteurs pour une littérature qui les fait trembler. Traductions et compilations de textes orientaux alimentent donc l'imaginaire fantastique entre 1720 et 1760. Les "récits d'élémentaires » (284) conçus autour de gnomes, de sylphes, d'ondins et de salamandres et auxquels $L e$ Comte de Gabalis de Montfaucon de Villars donne son coup d'envoi en 1670 font intervenir une part de burlesque. Dans la foulée de Villars, d'autres auteurs n'hésitent pas, au milieu du siècle des Lumières, à représenter une interaction avec des êtres surnaturels et donc à combiner le merveilleux et la réalité, projet auquel participent le thème du démembrement corporel et le motif de la tête coupée, dont traite longuement l'auteure. Ainsi, au fil du XVIII e siècle, le fantastique se définit moins par sa composante surnaturelle que par la mise à l'épreuve des personnages confrontés au surnaturel, c'est-à-dire par un « discours second, interrogatif, problématique » (347) face au pouvoir de l'imagination. 


\section{De l'inquiétude au fantastique}

À une époque où les structures sociales d'Ancien Régime sont menacées, le merveilleux classique souffre d' «évidement» (528), de sorte que l'inquiétude s'immisce dans le texte de fiction. La représentation de la mort, de la maladie et de la folie converge vers une angoisse charnelle, c'est-à-dire « directement vécue » (353). L'inquiétude liée au rapport au corps entraîne chez le lecteur ce que l'auteure qualifie de « convulsion » (355), c'est-à-dire de "réaction spontanée et violente produite par le contact avec l'étrangeté » (355). C'est alors que le fantastique se conçoit dans la confrontation du lecteur avec une expérience effrayante comme celle de la mort. Les discours encyclopédiques et médicaux qui passent en revue différents types de mort et qui compilent nombre de récits et de témoignages s'attardent surtout à la mort physique. Bien que la représentation de la mort donne dans la surenchère au sein du conte merveilleux, elle continue d'y être considérée dans ce qu'elle a de concret. Alors que les « cires anatomiques » (390) représentant le corps mort s'attirent un regard empreint à la fois d'étonnement et d'angoisse, figurines et statuettes jouent des rôles similaires dans les contes. Tandis que le dégoût qu'évoquent la puanteur et le verbe «vomir » dans les contes des années 1730 s'explique par l'inquiétude que provoque le corps d'autrui, le thème de l'impuissance qu'exploitent la sorcellerie et le libertinage manifeste plutôt l'angoisse qu'inspire son propre corps. Que la femme, la sorcière ou l'enchantement en soient la cause, l'impuissance est révélatrice de l'incompréhensible, réglant les mouvements et la souffrance du corps. Rêve, songe et cauchemar augmentent dans le texte 
de fiction le sentiment d'inquiétude et se présentent comme des symptômes du déséquilibre affectant le sujet dans son rapport au monde.

Dans cet ouvrage qui a l'avantage de faire découvrir des femmes écrivains peu étudiées telles que Mme Robert, Mme Murat, Mlle L'Héritier, Mme Lévesque et Mme d'Auneuil, Emmanuelle Sempère distingue judicieusement le "registre » fantastique des Lumières, conçu comme une «machine textuelle» (523) qui orchestre des représentations merveilleuses et surnaturelles et qui opère par le biais de la fascination et de la séduction, du genre fantastique qui se constituera au siècle suivant. De la superstition à l'inquiétude en passant par le surnaturel, le merveilleux et le fantasmagorique, cette étude montre le fantastique sous tous ses angles. 